Известия вузов России. Радиоэлектроника. 2021. Т. 24, № 4. С. 19-26 Journal of the Russian Universities. Radioelectronics. 2021, vol. 24, no. 4, pp. 19-26

Radio-Engineering Means of Transmission, Reception and Processing of Signals

UDC 621.391

Original article

https://doi.org/10.32603/1993-8985-2021-24-4-19-26

\title{
Improving the Structure of a Signal Used for Real-Time Calibrating of the Receiving Channels of Digital Transceiver Modules in Digital Phased Antenna Arrays
}

\author{
Viet Hung Tran ${ }^{凶}$, Minh Thien Hoang ${ }^{凶}$ \\ Le Quy Don Technical University, Hanoi, Vietnam \\ $\otimes$ hung.isi@lqdtu.edu.vn \\ $\bowtie$ thienhm.isi@lqdtu.edu.vn
}

\begin{abstract}
Introduction. Modern digital phased array antenna (DPAA) systems incorporate a large number of identical transceiver modules (TMs). These modules require real-time calibration with a high level of accuracy. In a previous work, we proposed a real-time calibration method for all receiver channels, which is based on the use of a calibration signal (CalSig) of the same frequency spectrum as the reflected signal and modulated in phase and amplitude by BPSK and OOK codes, respectively. This method was found to have a number of advantages over conventional approaches. However, the use of the same CalSig sample for all receiving channels increases the noise power gain at the output of a digital beam-forming unit (DBU). To overcome this limitation, we set out to improve the structure of CalSigs by making them pseudo-orthogonal. As a result, the noise power gain at the DBU output can be significantly reduced compared to that obtained in our previous work.

Aim. To propose an improved design of a controlled amplitude modulation code OOK generator, which allows creation of pseudo-orthogonal CalSigs. As a result, the noise power gain at the output will increase insignificantly, thus having no negative effect on the quality of digital beam forming, signal processing and calibration.

Materials and methods. Theory of system engineering and technology; theory of digital signal processing; system analysis; mathematical modeling.

Results. An improved CalSig for calibrating the receiving channels of TMs was obtained. A structural diagram allowing the formation of pseudo-orthogonal CalSigs was synthesized.

Conclusions. We proposed a new approach to improving the structure of signals used for real-time calibrating the DPAA receiving channels. A structural diagram of an amplitude-modulated OOK code generator for pseudoorthogonal CalSigs was developed.
\end{abstract}

Keywords: digital phased array antenna, real-time internal calibration, calibration signal, transceiver module, digital beamforming, integrated calibration subsystem

For citation: Viet Hung Tran, Minh Thien Hoang. Improving the Structure of a Signal Used for Real-Time Calibrating of the Receiving Channels of Digital Transceiver Modules in Digital Phased Antenna Arrays. Journal of the Russian Universities. Radioelectronics. 2021, vol. 24, no. 4, pp. 19-26. doi: 10.32603/1993-8985-2021-24-4-19-26

Conflict of interest. Authors declare no conflict of interest.

Submitted 17.06.2021; accepted 15.07.2021; published online 29.09.2021

Introduction. The method of real-time internal calibration of all receiver channels in digital phased antenna array (DPAA) systems is widely applied [16] for ensuring accuracy when forming a digital beam. Modern DPAA systems, as a rule, incorporate a large number of identical transceiver modules (TMs). Under the influence of such environmental factors as temperature or humidity and provided a high integration density of such TMs, the amplitude and phase parameters of a signal at the output of TM receiver channels may alter $[7,8]$. Thus, to ensure the required quality of digital beamforming and reflected signal processing, the receiving channels of TMs should undergo real-time calibration during operation. 
Известия вузов России. Радиоэлектроника. 2021. Т. 24, № 4. С. 19-26

Journal of the Russian Universities. Radioelectronics. 2021, vol. 24, no. 4, pp. 19-26

Fig. 1 presents a typical structural diagram of a DPAA system, in which the signal synthesizer performs the function of both generating a transmitting signal when transmitting and generating a calibration signal (CalSig) when receiving [4-6]. In order to make the system less cumbersome, high-frequency power dividers and high-frequency transmission lines are frequently used for transmitting signals to TMs. Therefore, the CalSigs fed to the input of the receiving channels can be considered essentially the same. Fig. 2 shows a structural diagram of a TM that integrates some components of a calibration subsystem for direct transmission of a signal to the receiving channel input. In comparison with conventional TM models, this scheme contains additional elements (marked yellow) in the receiving channel for calibration. This allows calibration to be performed in parallel with the receiving process.

In [9], we proposed a feasible method for internal calibration of receiving channels. Here, the CalSig structure is a signal that has the same frequency spectrum as the reflected signal, modulated in phase and amplitude according to BPSK and OOK codes and having a peak power equivalent to the internal noise

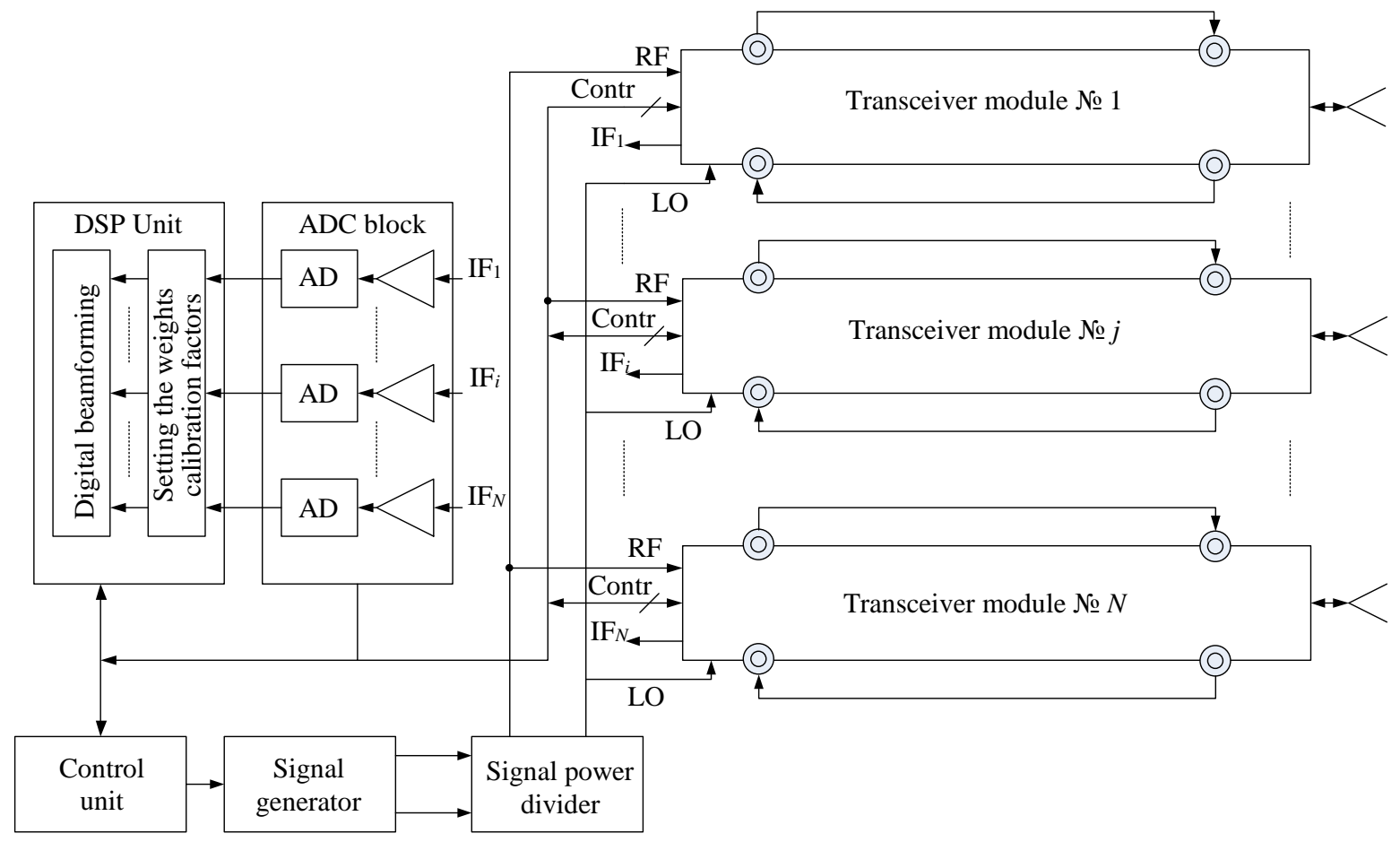

Fig. 1. Typical structure schema of DPAA system

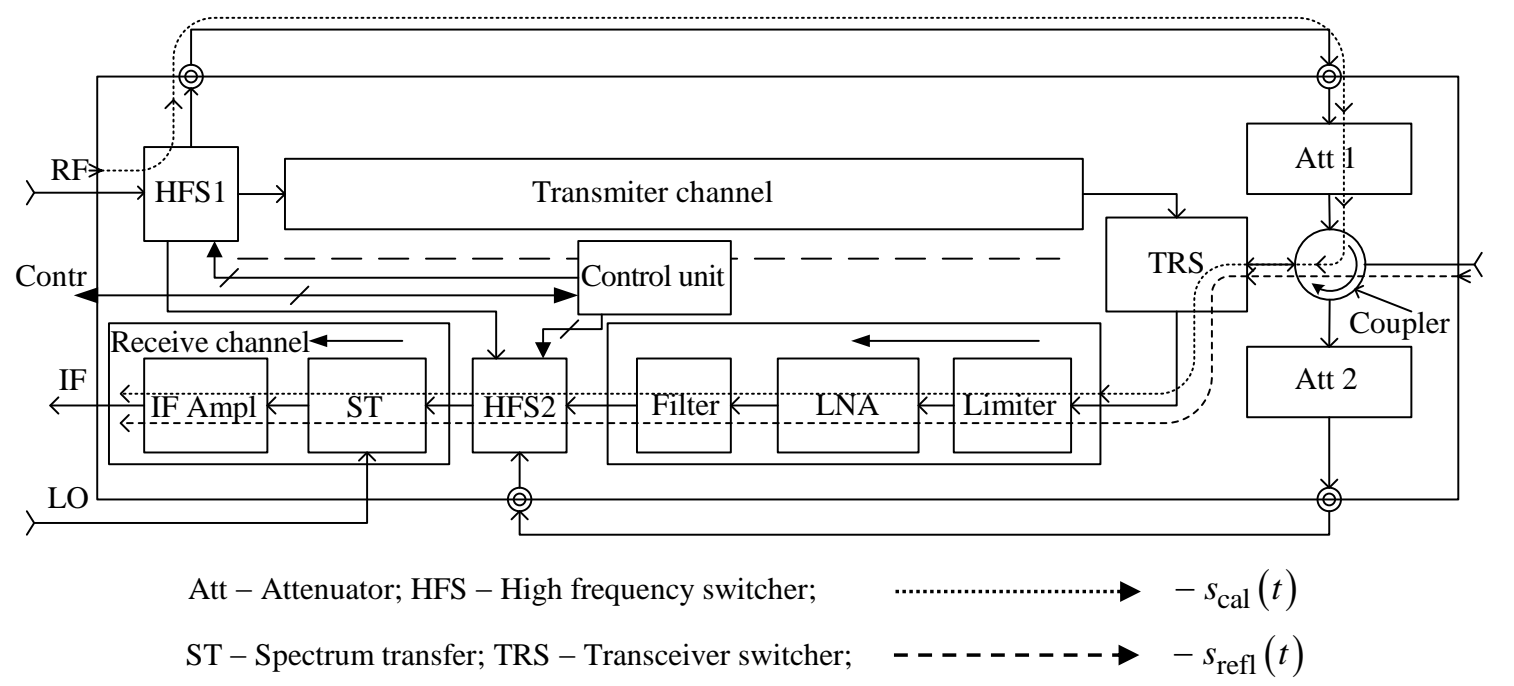

Fig. 2. Structure schema of a transceiver module 
Известия вузов России. Радиоэлектроника. 2021. Т. 24, № 4. С. 19-26 Journal of the Russian Universities. Radioelectronics. 2021, vol. 24, no. 4, pp. 19-26

power level of the receiving channel. Within the framework of this approach, the CalSig fed from outside to the input of the receiving channel can be treated as external noise during estimation calculations. Then, the external noise will combine with the internal noise to form a composite noise (referred to as noise). For example, a DPAA system has $N$ TMs calibrated by the same CalSig. Fig. 3, $a$ depicts the internal noise and the CalSig in the receiving channel. Let $P_{\mathrm{DBU}}$ be the gain of noise power at the DBU output, the CalSig have a peak amplitude level taken as the normalized internal noise level of "1", and the amplitude modulation by OOK code with the duty cycle $D$. Then, at the DBU output, the internal noise has a power of $N$ [10]. The CalSig has a peak amplitude of $N$, a peak power of $N^{2}$ and an average power of $N^{2} \times D$ (Fig. $\left.3, b\right)$. As a result, $P_{\mathrm{DBU}}$ will be equal to:

$$
\begin{gathered}
P_{\mathrm{DBU}} \approx \frac{N+N^{2} \times D}{N}=1+N \times D ; \\
P_{\mathrm{DBU}}[\mathrm{dB}]=10 \lg (1+N \times D) .
\end{gathered}
$$

Note that, since the internal noise level was normalized to "1", $P_{\mathrm{DBU}}$ will be considered as the noise power gain at the DBU output. From (1) and (2), $P_{\mathrm{DBU}}$ is proportional to the product of $N$ and $D$ in the positive direction. Therefore, since the number of TMs, $N$, is fixed, $P_{\mathrm{DBU}}$ can be reduced by reducing $D$. However, according to our previous conclusions [9], this contradicts the requirements for measurement time of errors and quality of calibration. The calculation and simulation results in MatLab showed that, if $D=1 / 45$ and $N=128$, the noise power gain $P_{\mathrm{DBU}}[\mathrm{dB}] \approx 5.8 \mathrm{~dB}$, which is too large for digital beamforming and signal processing. Thus, the approach to internal calibration of receiving channels presented in [9], although having advantages over conventional approaches, cannot be considered exhaustive.

In this paper, we aim to continue our research into improving the CalSig structure. The logic is as follows: establishing a set of pseudo-orthogonal CalSigs from the basic CalSig, leading to a very low correlation coefficient between them. This proposal is an improvement of the calibration method described in [9]. The research results are simulated in the MatLab environment.

Improving the CalSig structure. A structural scheme describing the formation of CalSigs. As shown in [9], we derived the phase and amplitude modulation from the basic CalSig $s_{\text {cal }}(n)$ by two code forms - BPSK and OOK. On their basis, $s_{\text {cal } i}(n)$ and $s_{\text {cal } j}(n)(i \neq j ; i, j \in N)$ can be obtained such that:

$$
K\left[s_{\mathrm{cal} i}(n), s_{\mathrm{cal} j}(n)\right] \rightarrow \min ,
$$

where $K\left[s_{\mathrm{cal} i}(n), s_{\mathrm{cal} j}(n)\right]$ is the correlation coefficient of $s_{\text {cal } i}(n), s_{\text {cal } j}(n)$. The ideal case is when $s_{\mathrm{cal} i}(n)$ and $s_{\mathrm{cal} j}(n)$ are orthogonal $[11,12]$. When the BPSK code is applied for phase modulation,

$$
C_{i}(n) \neq C_{j}(n) .
$$
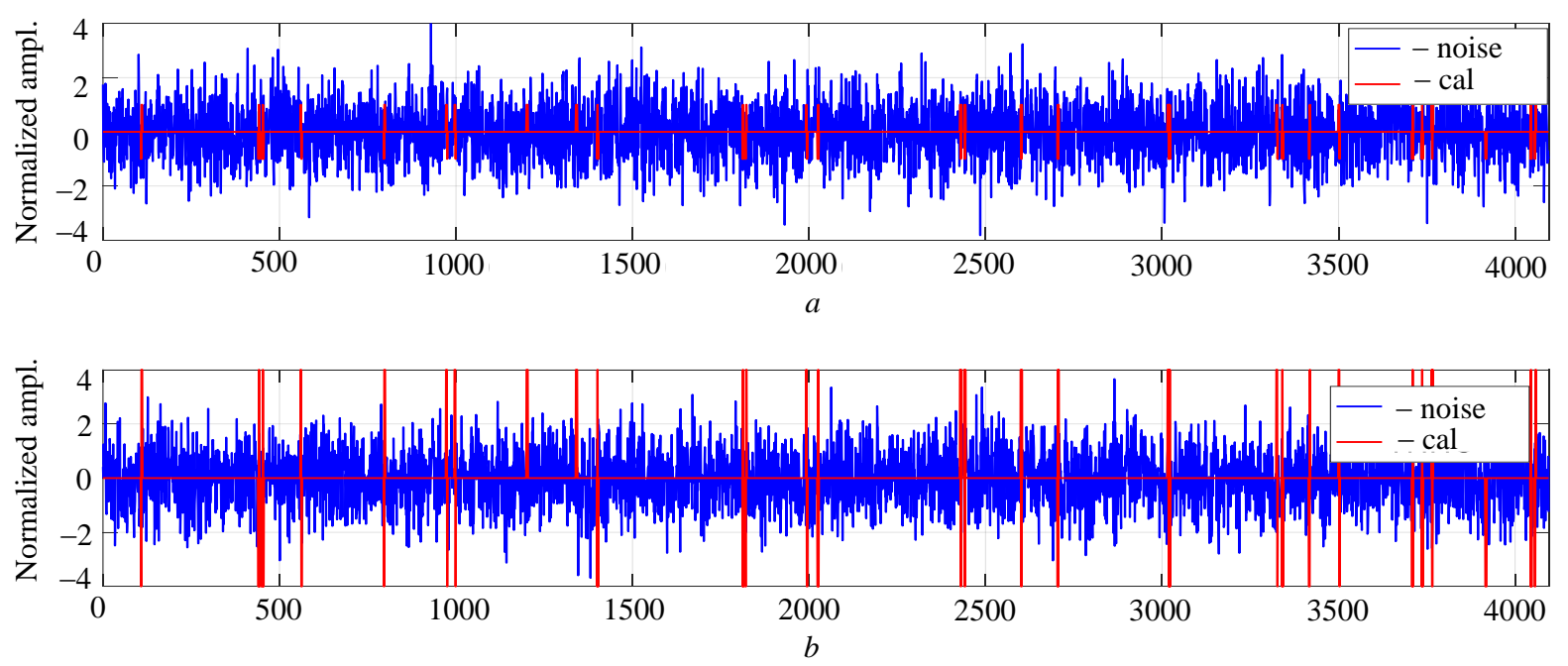

Fig. 3. Portrait of calibration signals and internal noise: $a$ - in a receiver channel; $b$-in the DBU 
Известия вузов России. Радиоэлектроника. 2021. Т. 24, № 4. С. 19-26

Then the structural scheme will have the form as shown in Fig. 4, a. Accordingly, to implement (3), phase shifter elements with control lines can be used. However, when the number of receiving channels $N$ is large, phase shift elements or flipping elements involved in hardware circuits will significantly complicate the entire system. Moreover, these elements may also become a source of phase and amplitude deviations [13].

The second option is to use OOK amplitude modulation codes [14] to form a CalSig for each receiving channel. Accordingly, the same OOK code will be used for generating $s_{\mathrm{cal} i}(n)$ and $s_{\mathrm{cal}} j(n)$, while the moment of the code activation control for each channel will be different. Then,

$$
\left.K_{\mathrm{cor}}\left[\operatorname{OOK}_{i}(n), \mathrm{OOK}_{j}(n)\right]\right|_{D} \rightarrow 0
$$

where $\left.K_{\text {cor }}\left[\operatorname{OOK}_{i}(n), \mathrm{OOK}_{j}(n)\right]\right|_{D}$ is the correlation coefficient between $\mathrm{OOK}_{i}(n)$ and $\mathrm{OOK}_{j}(n)$, provided that the duty cycle $D$ is the same for all receiving channels.

A structural scheme of this alternative is shown in Fig. $4, b$. Accordingly, the input signal will be the base signal $s_{\text {cal }}(n)$ that has been phase-modulated according to the BPSK code in the signal synthesizer. The results at the outputs will be

$$
s_{\text {cal } i}(n) \neq s_{\text {cal } j}(n),
$$

provided that $(i \neq j) \in N$.

An analysis of the two schemes in Fig. 4 shows that the second option is much simpler from the standpoint of engineering and technological design [15].

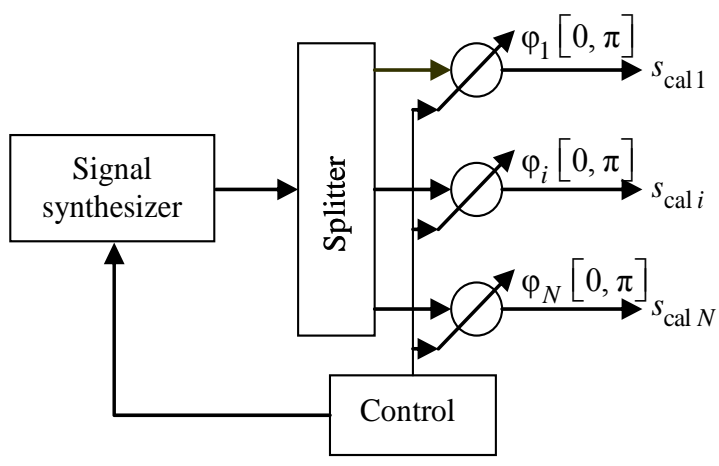

$a$
Fig. 5, $a$ and $b$ show the results of simulating the CalSig and the internal noise by two aforementioned options, provided that the duty cycle $D=1 / 32$ is the same. Fig. 5, $c$ shows the simulation results of the CalSig and the internal noise at the DBU output when $N=64$. A comparison of Fig. 3, $c$ and 5,c shows that, when $s_{\text {cal } i}(n)=s_{\text {cal } j}(n), K_{\text {cor }} \rightarrow 1$ (Fig. 3, $c$ ), the noise power gain $P_{\mathrm{DBU}}$ at the DBU output will be greater than that at $s_{\mathrm{cal} i}(n) \neq s_{\mathrm{cal} i}(n)$ (Fig. 5,c).

However, when the number of TMs is too large, the "ON" levels (equivalent to "1" ) in OOK amplitude modulation codes may overlap. As a result, the correlation coefficient $K_{\text {cor }}$ may not decrease to the desired value, and the noise power gain $P_{\mathrm{DBU}}$ will continue to affect the quality and accuracy of digital beamforming and signal processing. For greater clarity, the following section will estimate the $P_{\mathrm{DBU}}$ level and its impact on the detection quality of DPAA systems.

Internal noise gain at the DBU output and the CalSig impact on the performance quality of a radar system. Estimation of the internal noise power gain at the DBU output.

1. The procedure for estimating the power level of the noise gain at the $\mathrm{DBU} P_{\mathrm{DBU}}$ uncor is as follows:

- at the DBU output, the internal noise for a DPAA system consisting of $N$ TMs is taken as $N$, since the internal noise of a channel is normalized to "1" [10];

- the amplitude modulation OOK codes $\mathrm{OOK}_{i}(n)$ and $\mathrm{OOK}_{j}(n)$ are considered different for each receiving channel at $\forall i ; j \in N$. Hence, at the DBU output, all CalSigs can be considered as

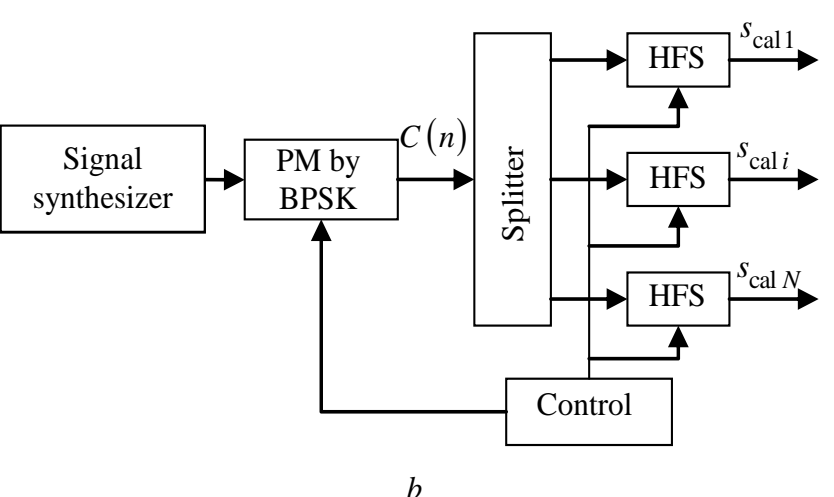

Fig. 4. Two schemes for the formation of pseudo-orthogonal calibration signals: $a$ - using phase shifters; $b$ - using switchs 
Известия вузов России. Радиоэлектроника. 2021. Т. 24, № 4. С. 19-26 Journal of the Russian Universities. Radioelectronics. 2021, vol. 24, no. 4, pp. 19-26
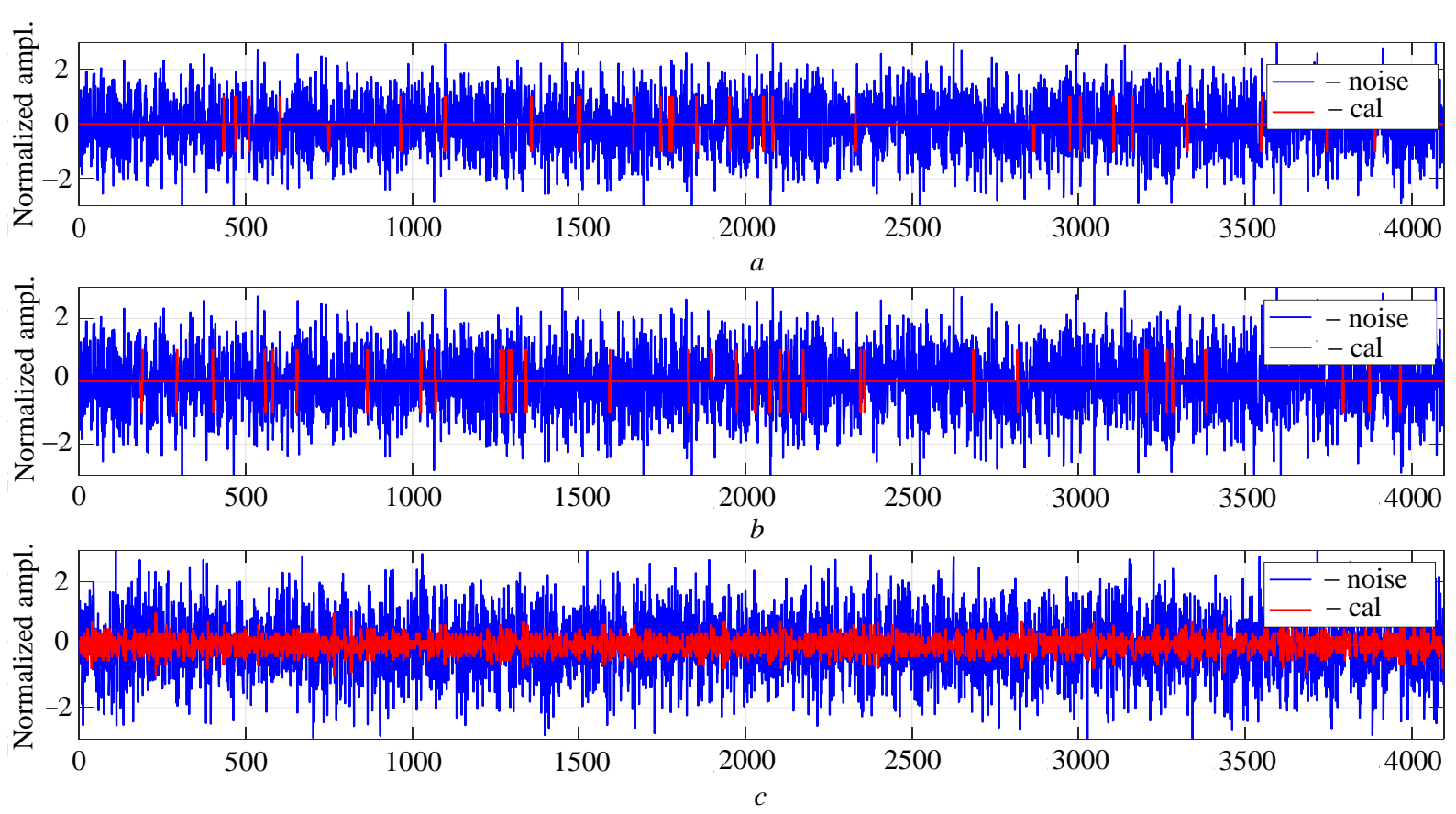

Fig. 5. Portrait of calibration signals and internal noise: $a, b$-when using OOK amplitude modulation code to form the calibration signals; $c$ - at the DBU

those evenly distributed over the time domain (Fig. 5,c).

The amplitude level of the CalSigs can be taken close to $N D$, with the average power level being equal to $(N D)^{2}$. The power level of the noise gain at the DBU output $P_{\mathrm{DBU}}$ uncor can be estimated as follows:

$$
P_{\mathrm{DBU} \text { uncor }} \approx \frac{N+(N D)^{2}}{N}=1+N \times D^{2},
$$

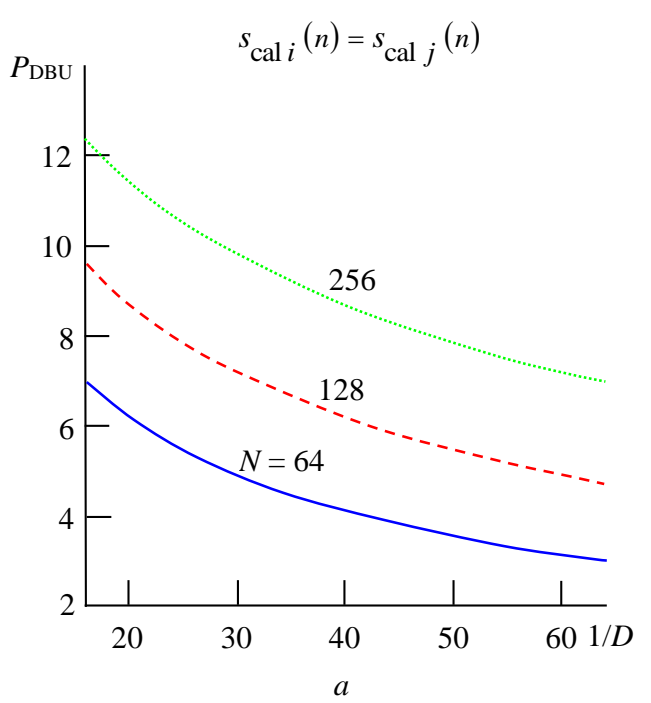

$$
P_{\mathrm{DBU} \text { uncor }}[\mathrm{dB}]=10 \lg \left(1+N \times D^{2}\right)=f(D) \text {. }
$$

A comparison of expressions (5) and (1) shows that

$$
P_{\mathrm{DBU} \text { uncor }} \ll P_{\mathrm{DBU}} \text {, }
$$

since the duty cycle $D$ is always lower than 1 .

Fig. 6 shows the power level of the noise power gain at the DBU output $P_{\mathrm{DBU}}$ and $P_{\mathrm{DBU} \text { uncor }}$ versus $D$ estimates with different numbers of TMs for the two cases: $\quad s_{\mathrm{cal} i}(n)=s_{\mathrm{cal} j}(n) \quad$ and

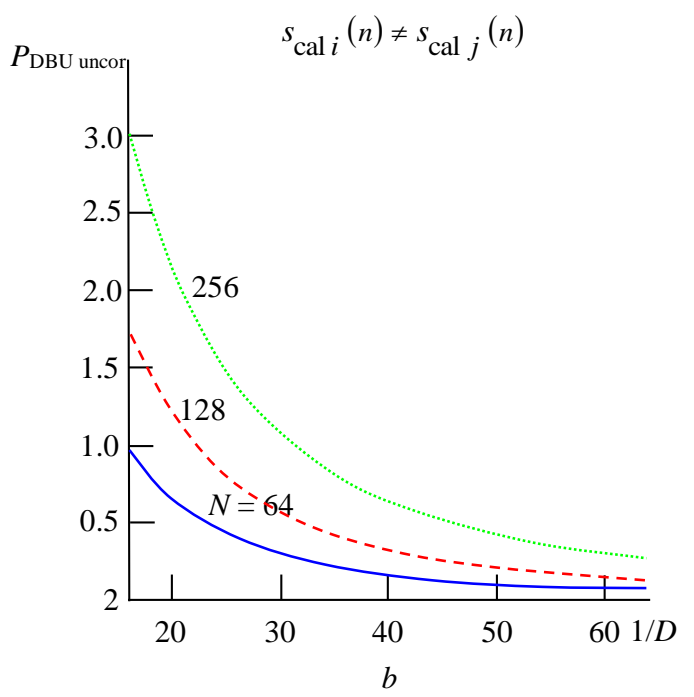

Fig. 6. Two graphs of the power level of the noise: $a$-when $s_{\mathrm{cal} i}(n)=s_{\mathrm{cal} j}(n) ; b-$ when $s_{\mathrm{cal} i}(n) \neq s_{\mathrm{cal} j}(n)$ 
Известия вузов России. Радиоэлектроника. 2021. Т. 24, № 4. С. 19-26

Journal of the Russian Universities. Radioelectronics. 2021, vol. 24, no. 4, pp. 19-26

$s_{\mathrm{cal} i}(n) \neq s_{\mathrm{cal} j}(n)$. For example, when the duty cycle $D=1 / 45$ and the number of TMs $N=128$, $P_{\mathrm{DBU}}[\mathrm{dB}] \approx 5.8 \mathrm{~dB}$ and $P_{\mathrm{DBU}}$ uncor $[\mathrm{dB}] \approx 0.27 \mathrm{~dB}$.

2. The simulation results also prove the validity of the presented approach. Fig. 7 shows an algorithm for simulating the determination of $P_{\mathrm{DBU}}$ uncor according to (4) with different values of $D$ and $N$ in the MatLab environment. Following this scheme, a continuous oscillating signal phase-modulated by the BPSK code is generated to form a basic CalSig $s_{\text {cal }}(n)$. For each receiving channel, the CalSig will be formed by multiplying $s_{\text {cal }}(n)$ by the OOK amplitude modulation code having a defined $D$. A normal-distributed internal noise is formed and added to each channel. Then, all the receiving channels are summarized and the power level of the noise gain at the DBU output is estimated. The simulation results are shown in Fig. 8.

Estimation of the CalSig impact on the performance quality of a radar system. Using the approach described in [9], the effect of CalSigs on the performance of a radar system should be estimated by considering the effect of the noise power gain at the DBU output on such an important parameter as the maximum detection distance for cases without and with a CalSig in each receiving channel. Accordingly, the relationship between the maximum detection distance and $P_{\mathrm{DBU} \text { uncor }}$ is expressed as follows [9]:

$$
R_{\max \text { Cal }} / R_{\max }=\sqrt[4]{1} / P_{\mathrm{DBU}} \text { unicor }
$$

Here, $R_{\max }$ and $R_{\max C a l}$ are maximum detection distances for cases without and with a CalSig, respectively.

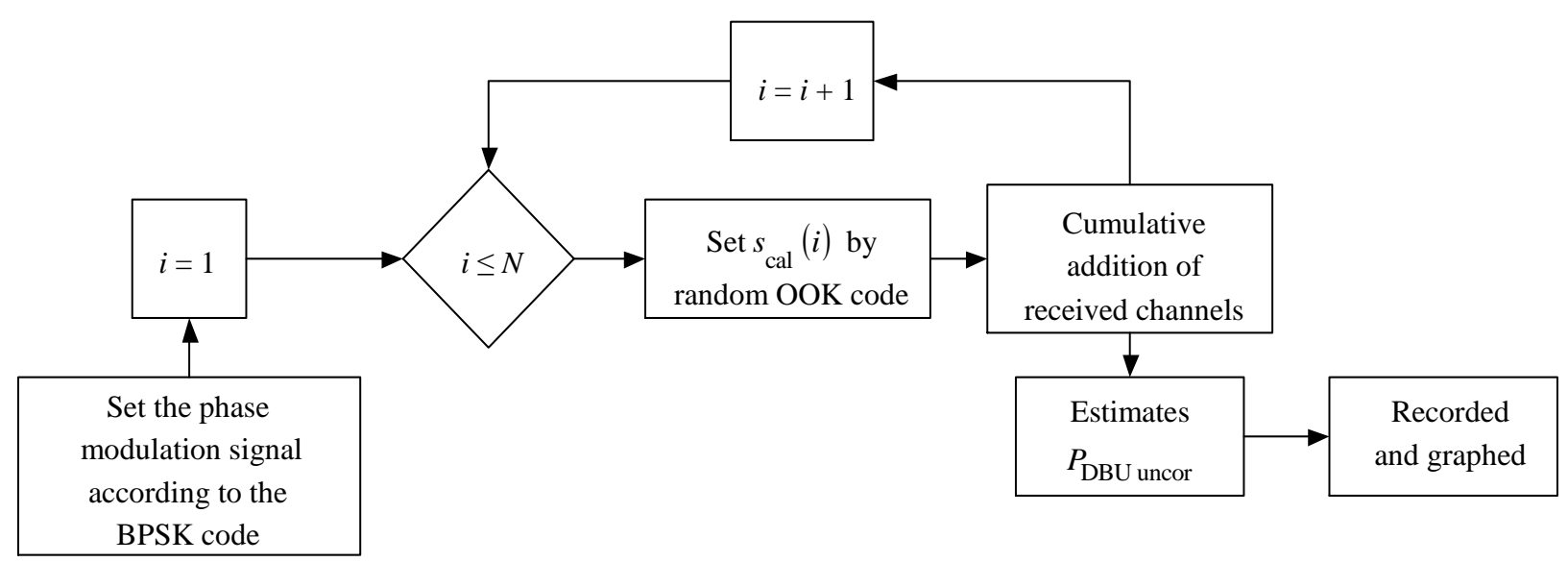

Fig. 7. Graph of the algorithm for simulating the determination of $P_{\mathrm{DBU}}$ uncor

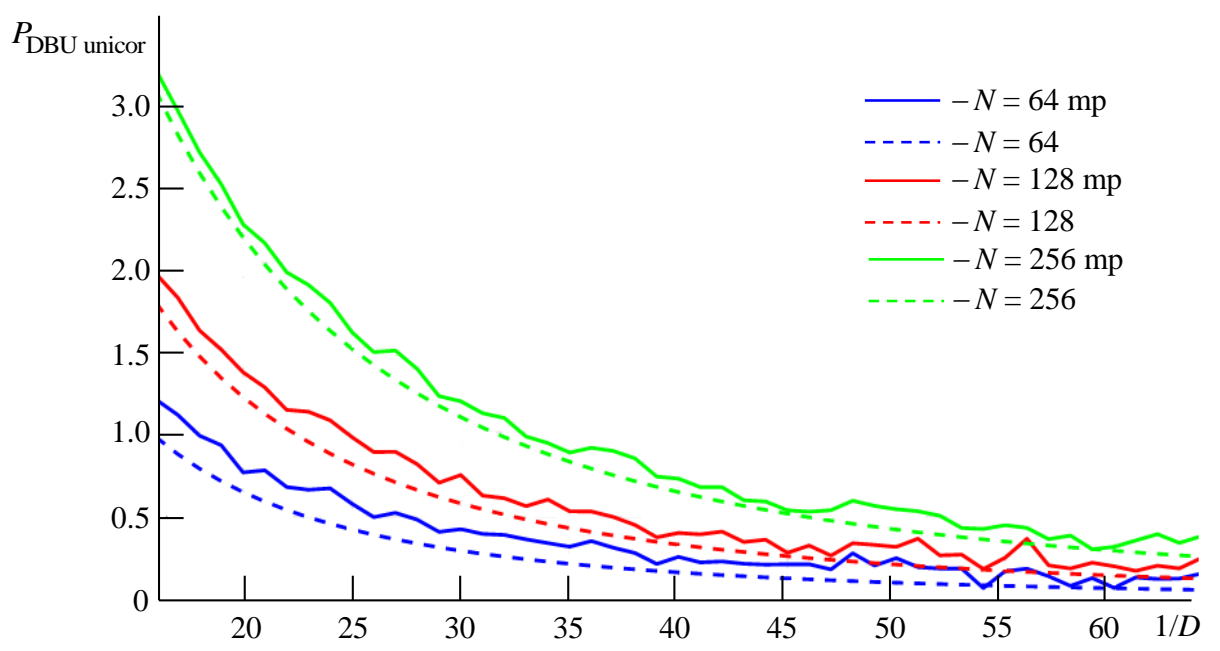

Fig. 8. Graph of simulation results of the noise power gain which is estimated at DBU 
It can be seen from (6) and Fig. 8 that CalSigs do affect the detection distance of a radar system. However, this effect will be lower when the CalSigs are not correlated or poorly-correlated due to $P_{\mathrm{DBU} \text { uncor }} \ll P_{\mathrm{DBU}}$. For example, to ensure $98 \%$ of the required operating distance with a permissible level of noise power gain at the DBU output $P_{\mathrm{DBU} \text { uncor }}[\mathrm{dB}] \sim 0.35 \mathrm{~dB}$ and a certain number of TMs $N=128$ or $N=256$, the duty cycle value equals $D=1 / 45$ or $1 / 60$, respectively. According to our approach described in [9], it is possible to verify whether the integrated calibration subsystem for internal calibration of receiving channels meets the real-time requirements of quality and accuracy.
Conclusion. This paper extends our previous research aimed at improving the structure of a signal used for internal calibration of the receiving TM channels in a DPAA system. It is proposed to form a pseudo-orthogonal CalSig for each receiving channel, which allows a significant reduction in the power level of the noise gain at the DBU output at the same time as ensuring the required quality of digital beamforming and signal processing. In addition, a structural diagram of a DPAA system with an integrated subsystem for automatically measuring and real-time calibrating the receiving channels of TMs was synthesized. The research results were simulated in the MatLab environment. The actual testing is being carried out at the Laboratory of the Institute of System Integration under LQD.TU SRVietnam.

\section{References}

1. Younis M., Rommelet T., de Almeida F. Q., Huber S., Martone M., Krieger G., Villano M. Investigations on the internal calibration of multi-channel SAR. 2017 IEEE Intern. Geoscience and Remote Sensing Symp. (IGARSS), 2017, pp. 5386-5389. doi: 10.1109/IGARSS.2017.8128221

2. Kim D.-Ch., Park S.-jin, Kim T.-W., Minz L., Park S.-O. Fully Digital Beamforming Receiver With a Real-Time Calibration for $5 G$ Mobile Communication. IEEE Transactions on Antennas and Propagation. 2019, vol. 67, no. 6, pp. 3809-3819. doi: 10.1109/TAP.2019.2902712

3. Hoffman J. P., Veilleux L., Perkovic D., Peral E., Shaffer S. Digital calibration of TR modules for real-time digital beamforming SweepSAR architectures. IEEE Aerospace Conf., Big Sky, USA, MT, 2012, pp. 1-8. doi: 10.1109/AERO.2012.6187084

4. Horst S. J., Hoffman J. P., Perkovic-Martin D., Shaffer S., Thrivikraman T., Yates Ph., Veilleuxet L. Implementation of RF circuitry for real-time digital beamforming SAR calibration schemes. Radar Systems (Radar 2012), IET Intern. Conf., pp. 1-6.

5. Hoffman J. P., Horst S., Perkovic D., Shaffer S., Ghaemi H., Veilleux L. Advances in digital calibration techniques enabling real-time beamforming SweepSAR architectures. IEEE Aerospace Conf., Big Sky, USA, MT, 2013. pp. 1-9. doi: 10.1109/AERO.2013.6497146

6. Hoffman J. P., Horst S., Veilleux L., Ghaemi H., Shaffer S. Digital calibration system enabling real-time on-orbit beamforming. IEEE Aerospace Conf., Big Sky, USA MT, 2014, pp. 1-11. doi: 10.1109/AERO.2014.6836218

7. Jens Reimann, Technique for Concurrent Internal Calibration during Data Acquisition for SAR Systems. Remote Sens. 2020, vol. 12(11), iss. 1773, pp. 1-9. doi: $10.3390 /$ rs12111773
8. Lin Y., Ma Q., Wang S., Bu X., An J. Calibration for spaceborne phased array antennas without interrupting satellite communications. Proc. $9^{\text {th }}$ WCSP, China, 2017, pp. 1-5. doi: 10.1109/WCSP.2017.8171093

9. Hung Tran Viet, Thien Hoang Minh. A real-time internal alibration method for radar systems using digital phase array antennas. INISCOM 2021, LNICST 379, pp. 88-103. doi: 10.1007/978-3-030-77424-0_8

10. Florian RUPPIN. Probability and Statistics Basic concepts. Available at: http://www.mit.edu/ ruppin/Lecture_ESIPAP_Ruppin_2018.pdf (accessed 15.09.2021)

11. Brautigam B., Schwerdt M., Bachmann M., Stangl M. Individual T/R module characterisation of the TerraSAR-X active phased array antenna by calibration pulse sequences with orthogonal codes. IEEE Intern. Geoscience and Remote Sensing Symp., 2007, pp. 5202-5205. doi: 10.1109/IGARSS.2007.4424034

12. Silverstein S. D. Application of orthogonal codes to the calibration of active phased array antennas for communication satellites. IEEE Transactions on Signal Processing. 1997, vol. 45, no. 1, pp. 206-218.

13. Surface Mount. Phase Shifter. JSPHS-23+. Available at: https://www.minicircuits.com/pdfs/JSPHS-23+.pdf (accessed 10.07.2021)

14. Yu-Tsung Lo, Chau-Chan Yui, Jean-Fu Kiang. OOK/BPSK-Modulated Impulse Transmitters Integrated With Leakage-Cancelling Circuit. IEEE Transactions on Microwave Theory and Techniques. 2013, vol. 61, no. 1, pp. 218-224. doi: 10.1109/TMTT.2012.2226746

15. Surface Mount. Switch. MSW-2-20+. Available at: https://www.minicircuits.com/pdfs/MSW-2-20+.pdf (accessed 10.07.2021) 


\section{Information about the authors}

Viet Hung Tran, an Engineer specializing in Radar Systems, 2010 at LQD TU, Hanoi, Vietnam, defended a Master's thesis in System Engineering and Control Automation, 2016, at the LQD TU SR Vietnam. A specialist in systems engineering, radio-electronic and radar technology. Area of expertise: microwave equipment and technology; radio-electronic and radar technology, systems engineering. An author of three scientific papers. Postgraduate student in radio electronic technology under the scientific supervision of Bao N. F.

Address: Technical University n. a. Le Qui Don, 236 Hoang Quoc Viet St., Hanoi, Vietnam

E-mail: tranviethung84@gmail.com

Minh Thien Hoang, an Engineer specializing in Radar Systems, 2005 at the LQD TU, Hanoi, Vietnam; defended a Master's thesis in Systems Engineering and Control Automation, 2008 at the LQD TU SR Vietnam; defended a doctoral dissertation in the specialty Microelectronic Technology at TU Electronics and Communication Tokyo, Japan (2015). Area of expertise: microwave technology and technology; radio electronic and radar technology; systems engineering; microelectronic technology; telecommunications. An author of six scientific papers.

Address: Technical University n. a. Le Qui Don, 236 Hoang Quoc Viet St., Hanoi, Vietnam

E-mail: thienhm.isi@lqdtu.edu.vn, nguyenphungbao@lqdtu.edu.vn; baonp@imc.org.vn

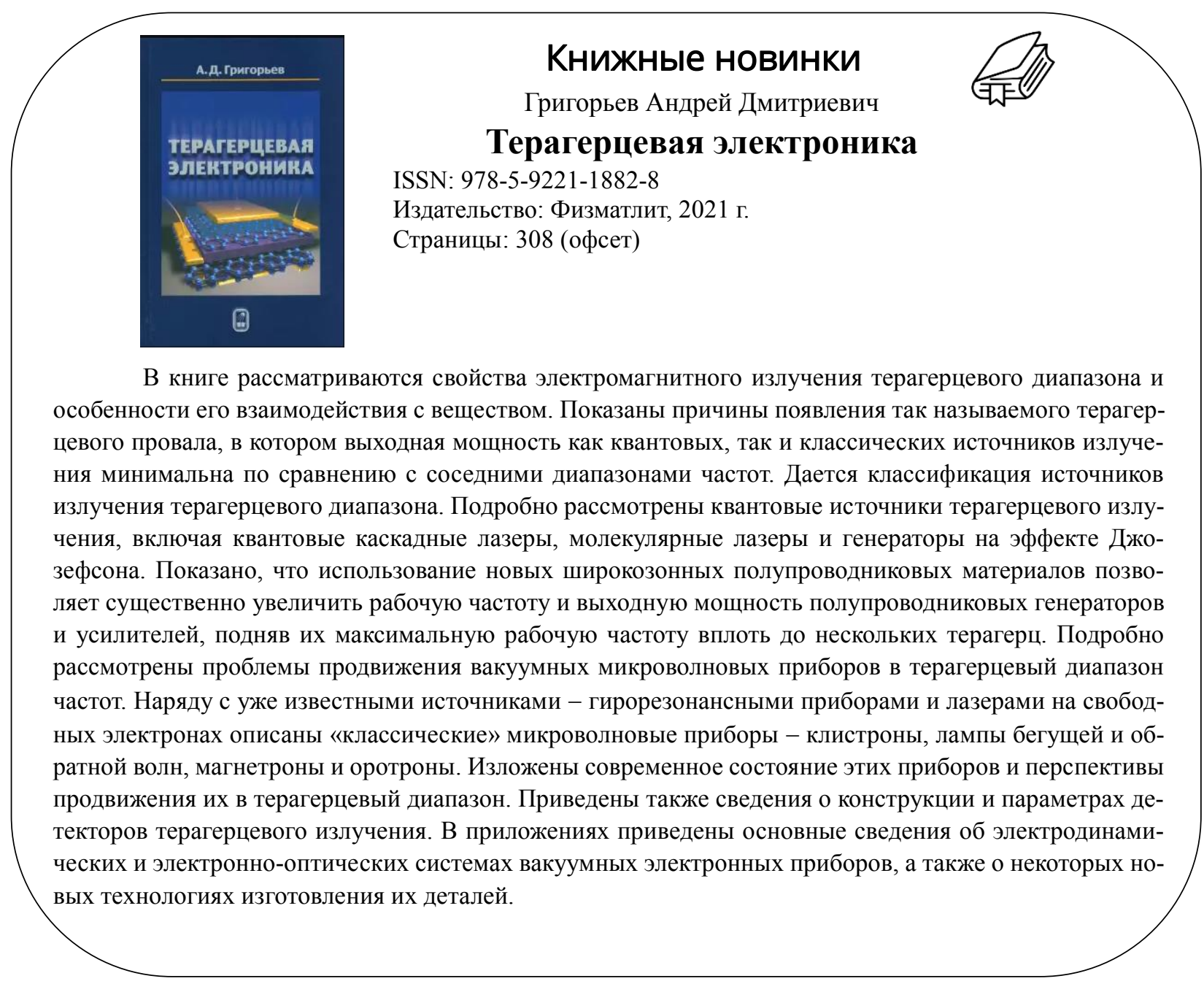

